\title{
Modelling nanoparticle transport in an animal exposure chamber: a comparison between numerical and experimental measurements
}

\author{
F. Morency \& S. Hallé \\ Département de Génie Mécanique, École de Technologie Supérieure, \\ Canada
}

\begin{abstract}
Nanoparticles transport in an exposure chamber is investigated using computational fluid dynamics (CFD). This exposure chamber is used to assess the lung toxicity in rats resulting from the inhalation of airborne NPs. The mathematical model for airflow is based on the three-dimensional Reynoldsaveraged Navier-Stokes equations with turbulence modelling. Simulations of airborne NPs are based on assumptions such that their motions are similar to the ones of a single sized diameter distribution of a passive contaminant.
\end{abstract}

Keywords: nanoparticles, CFD, exposure chamber, passive contaminant.

\section{Introduction}

The evaluation of the potential hazards for human health associated with inhalation or other form of contact with nanoparticles (NPs) is a question of much interest in the scientific community. Indeed, the phenomenal emergence of various types of nanotechnologies led many governmental agencies to present discussion papers on the safe use of nanometric scale particles, herein called NPs. These international reports are unanimous in supporting proactive measures to ensure the safety of workers exposed to NPs [1,2].

One of the basic elements for the risk assessment evaluation in a work environment consists in an adequate characterization of the degree of exposure. In the case of exposition to airborne NPs, numerical simulations could be used advantageously as a tool to safely predict these exposure levels. In order to achieve this though, the development of models is still required. It is expected that these models once validated will make it possible to predict the behaviour of airborne NPs in a workplace environment, to evaluate the effective exposure of 
humans to these NPs, and to safely assess the associated risks. This will in turn make it possible to design efficient ventilation and/or filtering systems which would make it possible to contain and to recover from an accidental release of undesirable NPs.

In that perspective of developing reliable models to simulate the transport and dispersion of airborne NPs, the objective of this paper is to present numerical results for a simple model of NPs dispersion in an animal exposure chamber. This exposure chamber will be used in further works to assess the lung toxicity in rats resulting from the inhalation exposures to airborne titanium dioxide $\left(\mathrm{TiO}_{2}\right)$ NPs.

More specifically, air flow simulation results are presented in the exposure chamber for which the NPs are assumed to behave as a passive contaminant. First, a complete description of the exposure chamber is provided. Then, the mathematical model for the air flow in the exposure chamber is detailed. Finally, some numerical results will be compared with experimental measurements made in the real chamber.

\section{Exposure chamber}

An exposure chamber is a device used to assess the toxicity of aerosols or gases during inhalation studies with laboratory animals. These inhalation studies, if carried out under controlled conditions, can simulate potential exposure conditions of humans at workplaces [3]. Controlled conditions mean that the level exposure must be uniform in space and time. The technical performance of our exposure chamber is critical for the credibility of an inhalation studies since non-uniform $\mathrm{TiO}_{2}$ concentration will lead to a non-uniform dose delivery to the rats.

Fig. 1 presents the experimental set-up that will be used for the in vivo study. The exposure chamber (Unifab, Kalamazoo, USA) is $0.5 \mathrm{~m}^{3}$ in volume. The chamber is equipped with 2 inlets located in the upper part and one outlet located

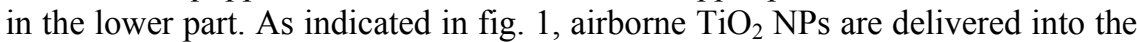
chamber by a 24 -jets Collison nebulizer. The aerosol, from inlet 2 , is then mixed and diluted by filtered fresh air admitted into the chamber via inlet 1 . A round deflector plate in the upper part ensures thorough and even distribution of air. In any case, the concentration of airborne NPs should be kept as uniform as possible in the zone delimited by the deflector at the top and the grid at the bottom. Such exposure rooms are usually design to work as a mixing chamber and therefore create a complex 3D air flow.

This exposure chamber is design for whole-body exposure studies. To adapt the actual chamber for a node-only system, seven holes were drilled on one side. These holes were tapped to receive each a $4.8 \mathrm{~cm}$ nominal Plexiglas pipe. The central tube is connected to a DUST Track and a Dekati sampling system (sampling rate $=201 / \mathrm{min}$ ). 


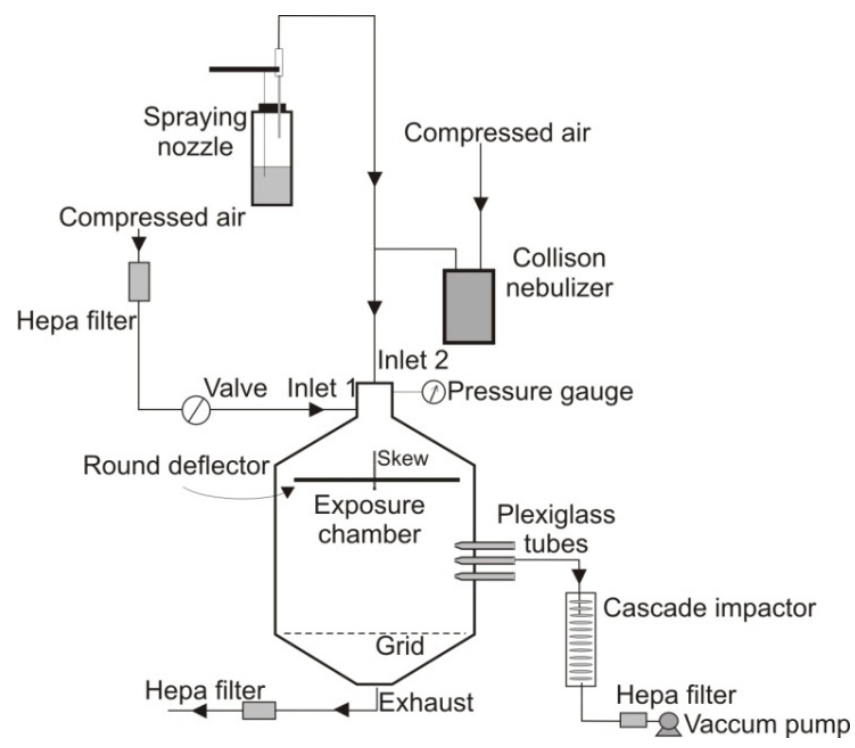

Figure 1: $\quad$ Schematic view of the experimental set-up.

\section{Mathematical model}

\subsection{Governing equations for the air flow}

Air flow within the exposure chamber is modelled by the three-dimensional Reynolds-averaged Navier-Stokes (RANS) equations using the eddy viscosity $\left(\vartheta_{t}\right)$ assumption. The mass conservation equation, eqn. (1), and the momentum conservation equation, eqn. (2), are:

$$
\begin{gathered}
\frac{\partial U_{i}}{\partial x_{i}}=0 \\
U_{j} \frac{\partial U_{i}}{\partial x_{j}}=-\frac{1}{\rho} \frac{\partial P}{\partial x_{i}}+\frac{\partial}{\partial x_{j}}\left[\left(\vartheta+\vartheta_{t}\right)\left(\frac{\partial U_{i}}{\partial x_{j}}+\frac{\partial U_{j}}{\partial x_{i}}\right)\right] .
\end{gathered}
$$

For the airflow studied in this paper, turbulent flow regime is present into the chamber. These turbulent effects are modelled by the $k-\omega$ turbulence model. In the $k$ - $\omega$ model, the eddy viscosity is function of the turbulent kinetic energy $(k)$ and the specific dissipation rate $(\omega)$. Both variables can be determined by solving two additional transport equations. More details on the $k-\omega$ turbulence model are available in Wilcox [4].

\subsection{Governing equations for the nanoparticles transport}

Airborne NPs are subjected to numerous physical phenomena that shape their size distribution in space and time. Coagulation leads to a reduction of the total number of particles, to an increase in the average particle diameter, and to an increase in the number of molecules in the particles. Growth of the particle 
occurs by gas-to-particle conversion. Sedimentation resulting from the gravity field also occurs, with the particle settling velocity depending on particle size.

However, under certain hypothesis, NPs distribution can be considered constant in space and time: i) for low NPs concentration, no coagulation or agglomeration of NPs will occur [5] and thus aerosol distribution is not altered; ii) for the exposure chamber, there is no gas-to-particle conversion; iii) sedimentation or settling of NPs is negligible in the global dispersion process, as current studies indicate $[6,7]$.

Moreover, because of their low concentration, NPs have no effects on the air flow and behave as a passive contaminant. This approximation is widely used for clean room design $[8,9]$.

In the exposure chamber, the generated NPs distribution is well represented by a single mean aerodynamic diameter. Several aerosol generation tests were performed by changing the input air pressure. The aerosol size distribution data obtained from a cascade impactor (Electrical Low Pressure Impactor, Dekati Inc.) located at inlet 2, show a maximum concentration of airborne $\mathrm{TiO}_{2}$ in the order of $9.40 \times 10^{6}$ particles $/ \mathrm{cm}^{3}$ with a relatively narrow size distribution around a mean aerodynamic diameter of $26 \mathrm{~nm}$ (fig. 2).

Under the assumptions stated above, the transport of a turbulent diffusing passive contaminant in a velocity field can be solved either using a Lagrangian approach, as in the studies of Wang et al. [10] and Horender et al. [11]. Another possibility is to solve an Eulerian model governed by the Reynolds averaged mass transport equation for the contaminant:

$$
\frac{\partial\left(\rho Z_{A}\right)}{\partial t}+\frac{\partial\left(\rho Z_{A} U_{j}\right)}{\partial x_{j}}=\frac{\partial}{\partial x_{j}}\left[\left(\rho D_{j}+\frac{\mu_{t}}{S c_{t}}\right)\left(\frac{\partial Z_{A}}{\partial x_{j}}\right)\right]
$$

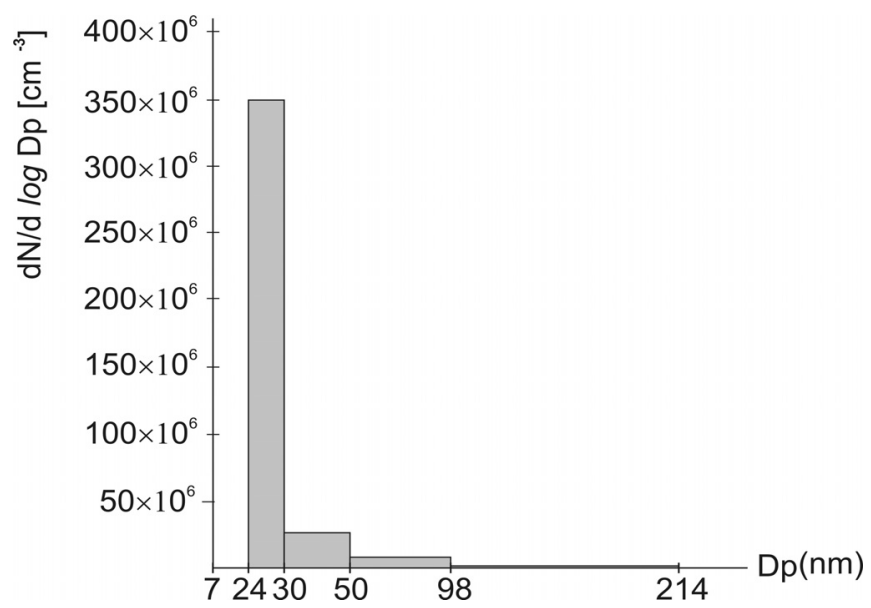

Figure 2: $\quad$ Experimental size distribution of airborne $\mathrm{TiO}_{2} \mathrm{NPs}$ at inlet 2 .

In eqn. (3), $Z_{\mathrm{A}}$ is the ratio of the contaminant mass fraction to the total mass, $D_{j}$ is the NPs Brownian diffusion coefficient and the turbulent Schmidt number 
$\left(\mathrm{Sc}_{\mathrm{t}}\right)$ represent the ratio of the eddy viscosity to the eddy diffusivity. The turbulence in the exposure chamber will generate diffusion effects which are orders of magnitude higher than Brownian diffusion. These turbulent diffusion effects are characterize by the turbulent Schmidt number which is usually close to unity; therefore $\mathrm{Sc}_{\mathrm{t}}$ was fixed to 0.9 . However, the intensity of turbulence becomes negligible near surfaces and the Brownian diffusion is the dominant mechanism in that region.

In a previous paper, Morency et al. [12], compared three Brownian diffusion models for NPs. They shown the models proposed by Friedlander [13] and Hinds [5] give almost identical diffusion coefficients while the diffusion coefficients obtain by the Gussman's model [14] are slightly lower. In the current study, $D_{j}$ was fixed to $6.0 \times 10^{-9} \mathrm{~m}^{2} / \mathrm{s}$ according to the value predicted by the Friedlander's model for a NP diameter of $30 \mathrm{~nm}$.

In the present study, the set of coupled eqns (1,2 and 3), are solved by a finite volume method using the commercial software FLUENT version 6.3. An Eulerian approach, similar to the one proposed here, has been used by other researchers such as Zhang et al. [15], to study NPs deposition in human tracheobronchial region or by Kumar et al. [16], to study NPs concentration in an urban street canyon.

\subsection{Computational domain and boundary conditions}

Fig. 3 shows a three-dimensional view of the exposure chamber computation domain. The model width $(\Delta \mathrm{x})$, length $(\Delta \mathrm{y})$ and height $(\Delta \mathrm{z})$ are $67.4 \mathrm{~cm}, 67.4 \mathrm{~cm}$ and $115.3 \mathrm{~cm}$ respectively. Diameter of the two inlets is $3.8 \mathrm{~cm}$. In order to limit

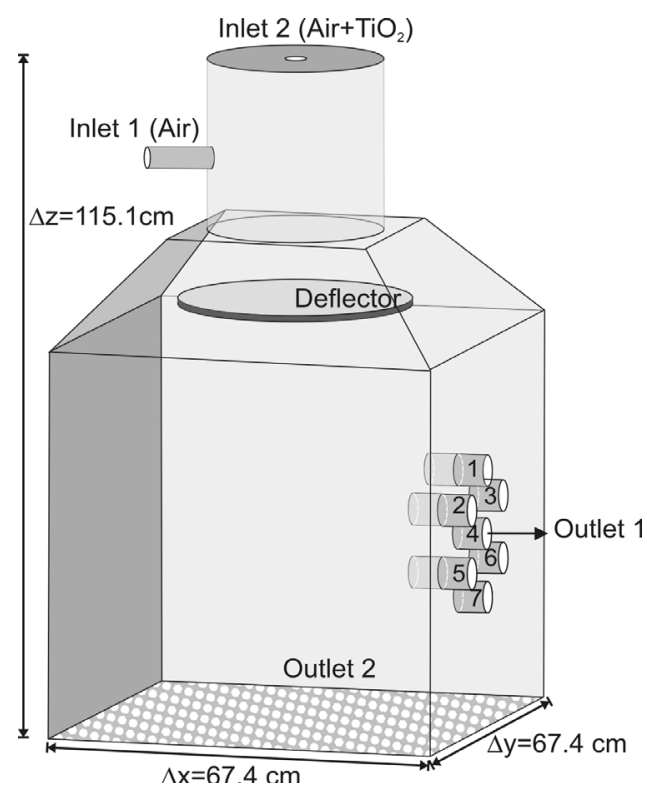

Figure 3: Computational domain 
the computational time, the bottom part of the exposure chamber was not considered in the numerical model. Therefore, the grid located at the bottom is modeled as an outlet vent in FLUENT. An outlet vent is an infinitely thin surface with a pressure drop proportional to the dynamic pressure of the flow. The velocity distribution at inlet 1 and 2 are considered uniform. No-slip conditions are imposed at wall for the velocity components.

The contaminant mass fraction to the total mass $\left(Z_{A}\right)$ imposed at inlet 2 is determined from the experimental size distribution of airborne $\mathrm{TiO}_{2} \mathrm{NPs}$. We have:

$$
Z_{A}=\frac{\left[n \times \pi / 6\left(D_{p}\right)^{3} \times \rho_{T i O 2}\right]}{\left[\text { total mass in } 1 \mathrm{~cm}^{3}\right]}
$$

with $n=9.40 \times 10^{6}$ particles $/ \mathrm{cm}^{3}, \rho_{\mathrm{TiO} 2}=3895 \mathrm{~kg} / \mathrm{m}^{3}$ (anatase $\mathrm{TiO}_{2}$ ) and $D_{p}=$ $26.4 \times 10^{-9} \mathrm{~m}$, the mass fraction at inlet 2 is set to $2.89 \times 10^{-7} \mathrm{~kg} / \mathrm{kg}$.

\subsection{Diffusional losses of nanoparticles}

Diffusion is the largest force acting on NPs in the laminar region of the boundary layer. Airborne NPs will disperse rapidly and will diffuse toward solid surfaces who act as a sink. Constant removal of NPs from the air by deposition on solid surfaces leads to significant diffusion losses in the exposure chamber.

It is not possible to develop an analytical expression to predict the loss of NPs for a complex three dimensional flow pattern such as the one observed in the exposure chamber. In a straight tube, NPs transport efficiency can be determined by the following relation [17]:

$$
\eta_{\text {diffusion }}=e^{-[\xi S h]},
$$

where $\xi=\frac{\pi D L}{Q}$. Key parameters determining the transport efficiency are the tube diameter $(D)$, the tube length $\mathrm{L}$, the Reynolds number based on flow rate $\mathrm{Q}\left(R e_{d}\right)$ and the particles diffusion coefficient $\left(D_{j}\right)$. For a tube with fully developed turbulent flow conditions, Friedlander [13] proposed a relation to determine the Sherwood number $(S h)$ :

$$
S h=0.0118 R e_{d}^{7 / 8}\left[\frac{\mu}{\rho_{a i r} D_{j}}\right]^{1 / 3} .
$$

NPs transport and diffusion in a turbulent pipe flow are calculated within FLUENT in order to compare the numerical results with Friedlander's relation. Fig. 4 presents the axisymmetric computational domain used in FLUENT. A constant velocity of $5 \mathrm{~m} / \mathrm{s}$ is imposed at inlet with a density of $1.225 \mathrm{~kg} / \mathrm{m}^{3}$, for $\mathrm{Re}_{\mathrm{d}}=3423$. For this flow condition, the entry length region spans less than $5 \%$ of the total pipe length.

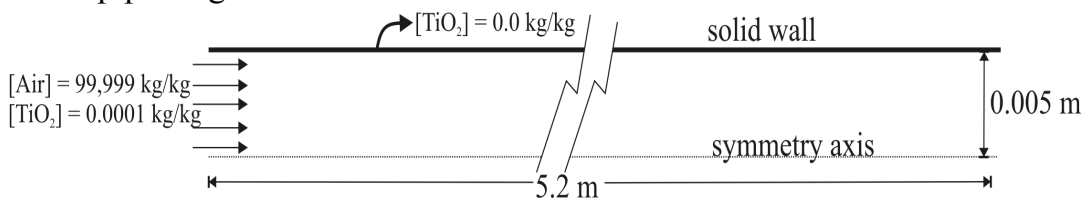

Figure 4: Tube flow geometry. 


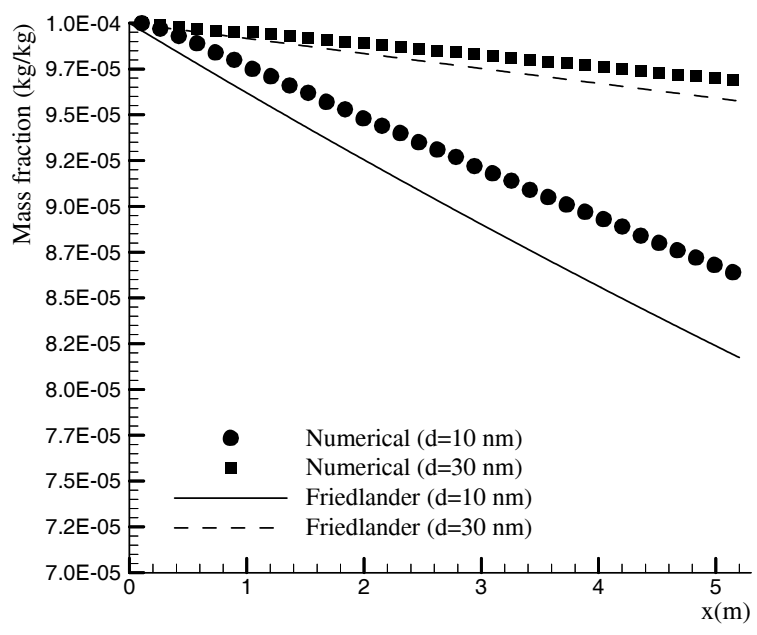

Figure 5: Mass fraction of airborne NPs in turbulent tube flow as a function of length.

Fig. 5 compares numerical transport efficiencies calculated by FLUENT to the ones obtained using eqn. (6). Results for NPs diameters of $10 \mathrm{~nm}$ and $30 \mathrm{~nm}$ are plotted. The numerical transport efficiencies are slightly higher than the correlation, around $6 \%$ of error in the worst case. The error band for the correlation is not known, but it can be expected to be similar to the one commonly admitted for heat transfer correlations, around $30 \%$.

\section{Results}

In this study, NPs were treated as a passive contaminant. This approximation is based on the hypothesis that the aerosol size distribution is not altered by coagulation. Fig. 6 presents the mass fraction distribution of NPs in the cylindrical part of the exposure chamber. Two-dimensional slice, at $y=0.0 \mathrm{~m}$, shows the mixing of fresh air from inlet 1 with airborne NPs from inlet 2. Initial mass fraction of $2.89 \times 10^{-7} \mathrm{~kg} / \mathrm{kg}$ at inlet 2 decreased to an average value of 1.0 $\times 10^{-7} \mathrm{~kg} / \mathrm{kg}$ at the cylinder bottom end $(\mathrm{z}=0.0 \mathrm{~m})$. Assuming spherical particle of $26 \mathrm{~nm}$ in diameter, this mass fraction correspond to $3.3 \times 10^{6}$ particles $/ \mathrm{cm}^{3}$. According to Hinds [5] for an initial concentration of $5 \times 10^{6}$ particles $/ \mathrm{cm}^{3}$, the time require to halve the number of particles by coagulation, is in the order of five minutes. With the actual ventilation rate of $143.5 \mathrm{l} / \mathrm{min}$, the NPs residence time is estimated to $3 \frac{1}{2}$ minutes, and it seems reasonable to neglect the coagulation of NPs as a first approximation. 


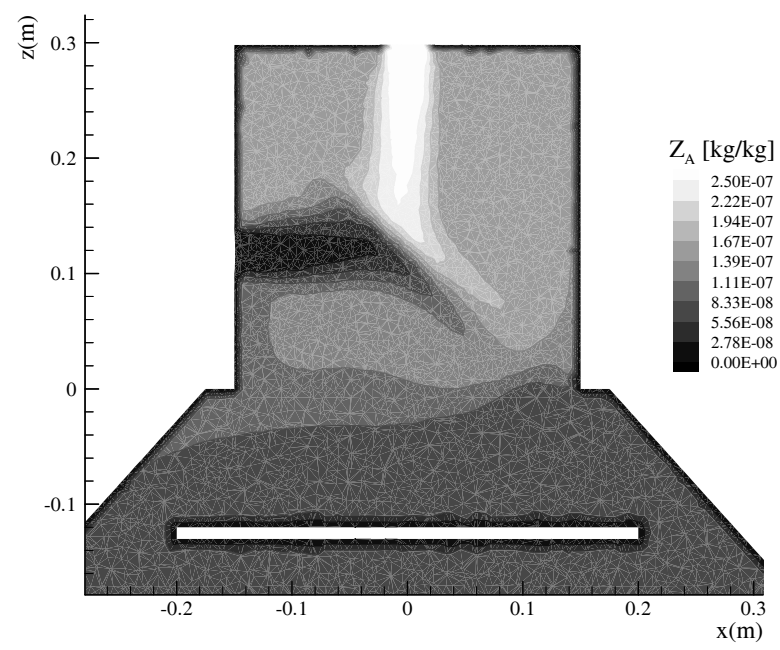

Figure 6: Mass fraction distribution in the cylindrical zone.

As stated in section 2, exposure rooms are design to work as a mixing chamber. Thus, the concentration of airborne NPs should be as uniform as possible in the animals breathing zone. Fig. 7 shows the two-dimensional mass fraction distribution in the Plexiglas tubes region. Predicted mass fraction present not significant variation in spite of the disturbances in the velocity distribution observe in Fig. 8. Central tube used for aerosol sampling created a suction zone with a velocity reaching $0.5 \mathrm{~m} / \mathrm{s}$ while the air velocity near tube 1 and 7 (Fig. 3) remain close to zero.

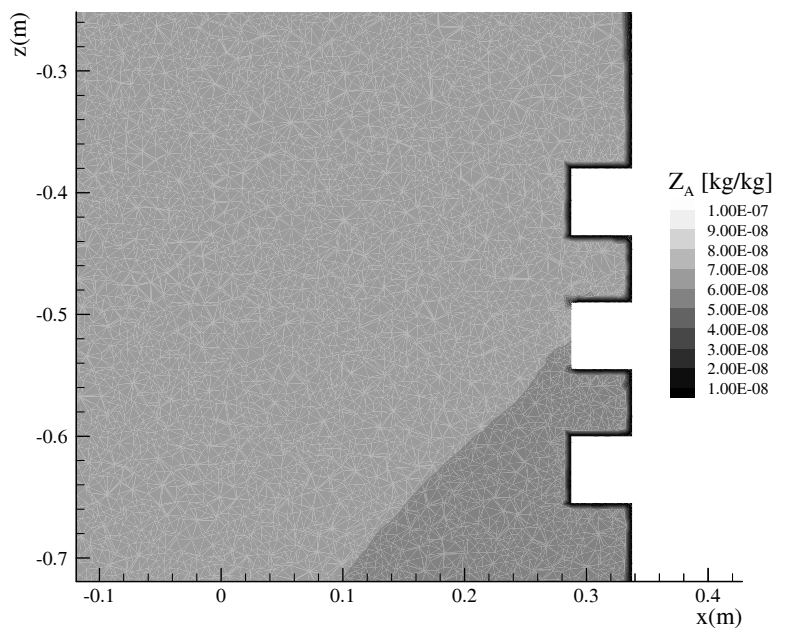

Figure 7: $\quad$ Mass fraction distribution in the breathing zone. 


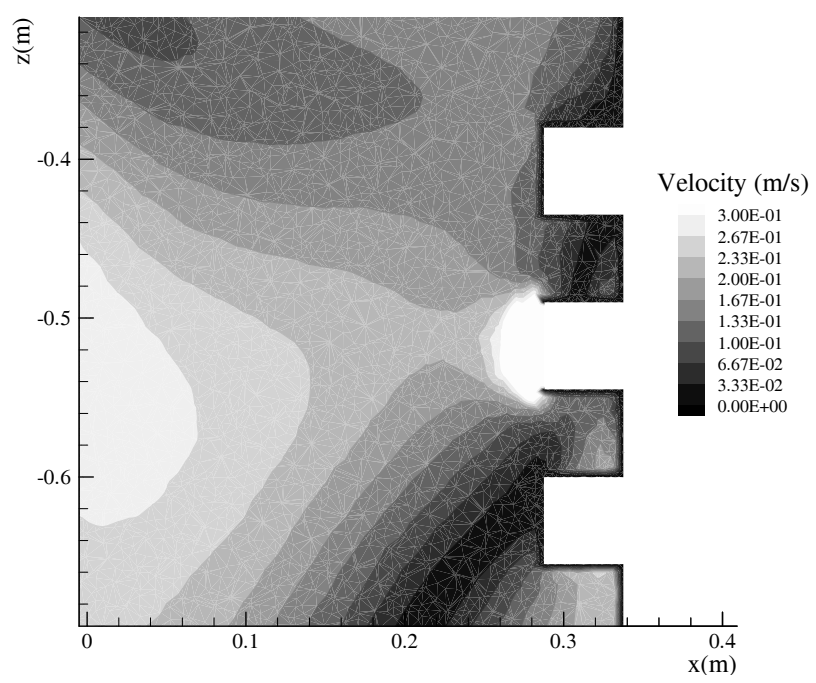

Figure 8: Velocity distribution in the breathing zone.

Table 1 presents the predicted mass fraction of NPs in the exposure chamber at seven location points. These seven points are respectively located on the centerline of each of the Plexiglas tube at $1 \mathrm{~cm}$ from the tubes tip. For the simulated ventilation and aerosol generation conditions, the NPs concentration in the rats breathing zone is fairly uniform. The averaged mass fraction is $6 \times 10^{-8}$ $\mathrm{kg} / \mathrm{kg}$ with a standard deviation of $0.13 \times 10^{-8} \mathrm{~kg} / \mathrm{kg}$. Table 1 also shows the measured mass fraction at the sampling tube (tube 4). Although the location of the Dekati sampling system probe is known approximately, the agreement between the experimental and predicted mass fraction is excellent.

Table 1: $\quad$ Comparisons of the predicted and measured mass fraction.

\begin{tabular}{|c|c|c|c|c|c|c|c|}
\hline \multicolumn{1}{|c|}{ Tube (Fig. 3) } & $\mathbf{1}$ & $\mathbf{2}$ & $\mathbf{3}$ & $\mathbf{4}$ & $\mathbf{5}$ & $\mathbf{6}$ & $\mathbf{7}$ \\
\hline $\begin{array}{l}\mathrm{Z}_{\mathrm{A}}\left(\times 10^{-8} \mathrm{~kg} / \mathrm{kg}\right) \\
\text { (numerical) }\end{array}$ & 6.12 & 6.15 & 5.90 & 6.02 & 6.04 & 5.80 & 5.92 \\
\hline $\begin{array}{l}\mathrm{Z}_{\mathrm{A}}\left(\times 10^{-8} \mathrm{~kg} / \mathrm{kg}\right) \\
\text { (measured) }\end{array}$ & & & & 5.59 & & & \\
\hline
\end{tabular}

\section{Conclusion}

This study focuses on airborne NPs transport and diffusion in an exposure chamber. Based on the assumption that NPs behave as a passive contaminant, the conclusions can be summarized as follows: 
a) Comparison between correlations and numerical simulations shown that diffusional losses of airborne NPs can be taken into account by imposing a zero mass fraction at solid walls.

b) The upper part of the exposure chamber act as a mixing chamber which create favorable flow conditions to disperse uniformly the NPs from inlet 2 and to prevent coagulation.

c) Mass fraction in the animal's breathing zone is uniform with a standard deviation at the seven Plexiglas tubes tips of only $2 \%$.

d) The agreement between the measured and predicted mass fraction at the sampling tube is remarkable.

\section{References}

[1] Aitken, R. J., Creely, K. S., \& Tran, C. L., Nanoparticles: An occupational hygiene review. Health \& Safety Executive Research Report 274, 2004.

[2] Ostiguy, C., Roberge, B., Menard, L., \& Endo, C.-A., Guide de bonnes pratiques favorisant la gestion des risques relies aux nanoparticules de synthèse. IRSST Guide technique R-586, 2008.

[3] Pauluhn, J., Overview of Inhalation Exposure Techniques: Strengths and Weakness. Experimental and Toxicologic Pathology, 57, pp. 111-128, 2005.

[4] Vorbau, M., Hillemann, L., \& Stintz, M., Method for the charcterization of the abrasion induced nanoparticle release into air from surface coatings. Aerosol Science, 40, pp. 209-217, 2009.

[5] Hinds, W. C., Aerosol Technology: Properties, Behavior, and Measurement of Airborne Particles. John Wiley \& Sons: New York, 1999.

[6] Lechner, M. D. \&Mächtle, W., Determination of the Particle Size Distribution of 5-100-nm Nanoparticles with the Analytical Ultracentrifuge: Consideration and Correction of Diffusion Effects. Progr. Colloid Polym. Sci., 113, pp. 37-43, 1999.

[7] Hervé-Bazin, B., Les nanoparticules - Une enjeu majeur pour la santé au travail? ed. INRS, EDP Sciences: Les Ulis, 2007.

[8] Hu, S. C., Chuah, Y. K., \& Yen, M. C., Design and Evaluation of a MiniEnvironment for Semi-Conductor Manufacture Processes. Building and Environment, 37, pp. 201-208, 2002.

[9] Zhao, B. \&Wu, J., Numerical Investigation of Particles Diffusion in a Clean Room. Indoor and Built Environment, 14(6), pp. 459-479, 2005.

[10] Wang, X., Gidwani, A., Girshick, S. L., \& McMurry, P. H., Aerodynamic focusing of nanoparticle: II. Numerical simulation of particle motion through aerodynamic lenses. Aerosol Science and Technology, 39(7), pp. 624-636, 2005.

[11] Horender, S., Lipowsky, J., Sommerfeld, M., Schwerin, M., \& Badeke, K.U., Deposition of $\mathrm{SiO} 2$ nanoparticles produced in a turbulent $\mathrm{H} 2 / \mathrm{O} 2$ Flame. Aerosol Science and Technology, 42(11), pp. 873-883, 2008. 
[12] Morency, F., Hallé, S., Dufresne, L., \& Emond, C., "Evaluation of Diffusion Models for Airborne Nanoparticles Transport and Dispersion," in Advances in Fluid Mechanics VII, Ashurst, England, 2008.

[13] Friedlander, S. K., Smoke, Dust, and Haze - Fundamentals of Aerosol Dynamics. Oxford University Press: New York, 2000.

[14] Gussman, R. A., On the Aerosol Particle Slip Correction Factor. J. Appl. Meteorol., 8, pp. 999-1001, 1969.

[15] Zhang, Z., Kleinstreuer, C., \& Kim, C. S., Airflow and nanoparticle deposition in a 16-generation tracheobronchial airway model. Annals of Biomedical Engineering, 36(12), pp. 2095-2110, 2008.

[16] Kumar, P., Garmory, A., Ketzel, M., Berkowicz, R., \& Britter, R., Comparative study of measured and modelled number concentrations of nanoparticules in an urban street canyon. Atmospheric Environment, 43, pp. 949-958, 2009.

[17] Brockman, J. E., Sampling and transport of aerosol, Aerosol measurements: principles, techniques and applications. 2nd edition, eds. P. A. Baron and K. Willeke, Wiley-InterScience Inc.: New-York, 2001. 